\title{
Nonlinear symmetric stability of planetary atmospheres
}

Article

Published Version

Bowman, J. C. and Shepherd, T. G. (1995) Nonlinear symmetric stability of planetary atmospheres. Journal Of Fluid Mechanics, 296. pp. 391-407. ISSN 0022-1120 doi: https://doi.org/10.1017/S0022112095002175 Available at https://centaur.reading.ac.uk/32868/

It is advisable to refer to the publisher's version if you intend to cite from the work. See Guidance on citing.

Published version at: http://dx.doi.org/10.1017/S0022112095002175

To link to this article DOI: http://dx.doi.org/10.1017/S0022112095002175

Publisher: Cambridge University Press

All outputs in CentAUR are protected by Intellectual Property Rights law, including copyright law. Copyright and IPR is retained by the creators or other copyright holders. Terms and conditions for use of this material are defined in the End User Agreement.

\section{www.reading.ac.uk/centaur}

\section{CentAUR}

Central Archive at the University of Reading

Reading's research outputs online 


\title{
Nonlinear symmetric stability of planetary atmospheres
}

\author{
By JOHN C. BOWMAN† AND THEODORE G. SHEPHERD \\ Department of Physics, University of Toronto, Toronto M5S 1A7 Canada
}

(Received 24 March 1995)

The energy-Casimir method is applied to the problem of symmetric stability in the context of a compressible, hydrostatic planetary atmosphere with a general equation of state. Formal stability criteria for symmetric disturbances to a zonally symmetric baroclinic flow are obtained. In the special case of a perfect gas the results of Stevens (1983) are recovered. Finite-amplitude stability conditions are also obtained that provide an upper bound on a certain positive-definite measure of disturbance amplitude.

\section{Introduction}

A classical problem in geophysical fluid dynamics is that of symmetric stability. The adjective 'symmetric' implies that both the equilibrium flow and allowed perturbations are independent of one coordinate. Symmetric instability is a kind of baroclinic instability that can occur even when the atmosphere is both statically and inertially stable (positively stratified with a monotonically increasing circulation). It has primarily been considered in the context of two physical applications: (i) mesoscale dynamics and (ii) planetary circulations. Sometimes referred to as slantwise convection, this instability produces symmetric convective rolls that have been identified with frontal rainbands in the mesoscale context and with axisymmetric cells in the planetary context.

Symmetric stability criteria were first derived by Fjørtoft (1950). These criteria have recently been extended to finite amplitude in the case of the $f$-plane Boussinesq equations - relevant to the mesoscale context - by Cho, Shepherd \& Vladimirov (1993), using the so-called energy-Casimir stability method. This method relies on the construction of a positive-definite invariant for disturbances to a given equilibrium, using the conservation of total energy together with the material conservation of entropy and angular momentum.

The present work extends the results of Cho et al. (1993) to planetary circulations, including the effects of both spherical geometry and compressibility, in terms of a general equation of state. Beyond these additional physical effects, certain novel mathematical features are exhibited in the analysis: multi-valued inverse equilibrium profiles and a moving domain of integration. The latter leads to some technical difficulties with convexity.

The equations describing the compressible hydrostatic model, which assumes zonal (azimuthal) symmetry and neglects diabatic processes, are presented in $\S 2$. It is shown that the total energy and certain functionals of the angular momentum, entropy, and

$\dagger$ Present address: Institute for Fusion Studies, University of Texas, Austin, TX 78712, USA. 
potential vorticity are conserved in time. In $\S 3$, we demonstrate that a general steady baroclinic zonal flow satisfying thermal-wind balance constitutes an equilibrium of the model system and corresponds to a conditional extremal of the total energy. In $\S 4$, we examine the formal stability of these baroclinic flows and obtain conditions equivalent to the classical criteria of inertial stability, static stability, and positivity of the product of the Coriolis parameter and the potential vorticity. In the special case of a perfect (ideal) gas, these criteria reduce to those of Stevens (1983). A finite-amplitude extension of this result, based on convexity arguments, is described in \$5. Although we are not able to prove Liapunov stability, we do derive a rigorous upper bound on disturbance amplitude that is expressible solely in terms of initial amplitudes, with the bound going to zero as the initial disturbance tends to zero. We find that the finite-amplitude criteria imply formal stability and are expressible as generalizations of the formal symmetric stability conditions.

\section{Governing equations}

We consider the model of hydrostatic compressible flow known in meteorology as the primitive equations. Denoting the latitude by $\lambda$, the longitude by $\varphi$ and the pressure by $p$, we describe a longitudinally symmetric spherical shell at radius $r=$ $a$, of thickness $\Delta r \ll a$, rotating at constant angular velocity $\Omega$. Using pressure coordinates $(\lambda, \varphi, p)$ with corresponding velocities $(u, v, \omega)$, the model equations for axisymmetric flow are (e.g. see Lorenz 1967):

$$
\begin{gathered}
\frac{\mathrm{D} u}{\mathrm{D} t}-\frac{\tan \varphi}{a} u v-2 \Omega v \sin \varphi=0, \\
\frac{\mathrm{D} v}{\mathrm{D} t}+\frac{\tan \varphi}{a} u^{2}+2 \Omega u \sin \varphi=-\frac{1}{a} \Phi_{\varphi}, \\
\frac{1}{a \cos \varphi} \frac{\partial}{\partial \varphi}(v \cos \varphi)+\frac{\partial \omega}{\partial p}=0, \\
\frac{\mathrm{D} s}{\mathrm{D} t}=0, \quad \Phi_{p}=-\frac{1}{\rho} .
\end{gathered}
$$

Here $\rho$ is the fluid density, $s$ is the specific entropy, and $\Phi \doteq g r$ is the geopotential, where the gravitational acceleration $g$ is treated as a constant. (We emphasize definitions with the notation '..') The upper surface is taken to be an isobaric surface on which, for simplicity, we suppose that $p$ approximately vanishes. (This effectively requires that the density scale height be much less than the thickness of the shell: $\left(\rho^{-1} \partial \rho / \partial r\right)^{-1} \ll \Delta r$. $)$ The appropriate boundary conditions are then $\omega=0$ on the upper surface and $\mathrm{D} \Phi / \mathrm{D} t=0$ on the lower surface (on which $r=a$ ), where the material or Lagrangian derivative $\mathrm{D} / \mathrm{D} t$ is given by

$$
\frac{\mathrm{D}}{\mathrm{D} t} \doteq \frac{\partial}{\partial t}+\frac{v}{a} \frac{\partial}{\partial \varphi}+\omega \frac{\partial}{\partial p} .
$$

Equation (2.1a) implies the material conservation of the axial component of the absolute angular momentum

$$
m(\varphi, p) \doteq u(\varphi, p) a \cos \varphi+\Omega a^{2} \cos ^{2} \varphi,
$$

namely, that $\mathrm{D} m / \mathrm{D} t=0$. 
It is common in meteorology to formulate results in terms of the potential temperature

$$
\theta(s) \doteq \exp \left(\int \frac{\mathrm{d} s}{c_{p}\left(s, p_{r}\right)}\right),
$$

instead of the entropy, where $c_{p}>0$ is the specific heat at constant pressure and $p_{r}$ is a fixed but arbitrary reference pressure. However, the stability analysis in this work is most naturally formulated in terms of a generalized potential temperature $\eta=\eta(s)$ defined by

$$
\eta \doteq \int \theta \mathrm{d} s=\int c_{p} \mathrm{~d} \theta
$$

For a fluid with constant $c_{p}, \eta$ is proportional to $\theta$. In the general situation, $\eta$ appears to be the optimal variable in which to cast the stability analysis because the second derivative of the enthalpy

$$
h(\eta, p) \doteq \mathscr{E}(\eta, p)+\frac{p}{\rho(\eta, p)}
$$

with respect to this variable vanishes. Here $\mathscr{E}(\eta, p)$ is the internal energy and $\rho=\rho(\eta, p)$ is the equation of state. From the Maxwell relation

$$
\mathrm{d} h=T \mathrm{~d} s+\frac{1}{\rho} \mathrm{d} p
$$

we see that

$$
h_{\eta}=\frac{T}{\theta}, \quad h_{p}=\frac{1}{\rho},
$$

where $T=T(\eta, p)$ is the temperature. The enthalpy is assumed to have continuous second derivatives so that $T$ and $\rho$ are related by

$$
\frac{T_{p}}{\theta}=\left.\frac{\partial}{\partial p}\right|_{\eta}\left(\frac{T}{\theta}\right)=\left.\frac{\partial}{\partial \eta}\right|_{p}\left(\frac{1}{\rho}\right)=-\frac{\rho_{\eta}}{\rho^{2}} .
$$

The thermodynamic relation

$$
\left.\frac{\partial T}{\partial s}\right|_{p}=\frac{T}{c_{p}}
$$

and (2.5) imply that the second derivative of $h$ with respect to $\eta$ vanishes,

$$
h_{\eta \eta}=\left(\frac{T}{\theta}\right)_{\eta}=\frac{T}{c_{p} \theta^{2}}-\frac{T \theta_{\eta}}{\theta^{2}}=0
$$

It is also convenient to introduce the potential vorticity $q \doteq \rho^{-1} \boldsymbol{Z} \cdot \nabla \theta$, where in the shallow-layer approximation

$$
Z \doteq \nabla \times(u \hat{\hat{\lambda}})+f \hat{r}=\left(\frac{u}{a} \tan \varphi-\frac{1}{a} u_{\varphi}+2 \Omega \sin \varphi\right) \hat{r}+\left.\frac{\partial u}{\partial r}\right|_{\varphi} \hat{\varphi}
$$

is the absolute vorticity associated with an axisymmetric flow. In terms of the radial component of $\boldsymbol{Z}$,

$$
Z \cdot \hat{\boldsymbol{r}}=\frac{u}{a} \tan \varphi-\frac{1}{a} u_{\varphi}+2 \Omega \sin \varphi=-\frac{m_{\varphi}}{a^{2} \cos \varphi},
$$


one may express $q$ as

$$
q=-\frac{g}{c_{p}}\left(Z \cdot \hat{r} \eta_{p}+\frac{1}{a} u_{p} \eta_{\varphi}\right)=\frac{g}{c_{p} a^{2} \cos \varphi} \frac{\partial(m, \eta)}{\partial(\varphi, p)} \equiv \frac{1}{\rho a \cos \varphi} \hat{\lambda} \cdot \nabla \theta \times \nabla m .
$$

Using the Lagrangian invariance of $m$ and $\eta$ together with (2.1c), (2.14) may be used to show that $\mathrm{D} q / \mathrm{D} t=0$.

The total energy for a spherical shell (of volume $V$ ) of this fluid is given by

$$
\overline{\mathscr{H}} \doteq \int_{-\pi / 2}^{\pi / 2} \int_{a}^{a+\Delta r(\varphi, t)} \rho\left(\frac{u^{2}+v^{2}}{2}+\mathscr{E}+\Phi\right) a^{2} \cos \varphi \mathrm{d} r \mathrm{~d} \varphi .
$$

Upon using the hydrostatic relation and integrating by parts (taking $p=0$ on the upper surface), we may rewrite $\overline{\mathscr{H}}$ in pressure coordinates:

$\overline{\mathscr{H}}\left\{m, v, \eta, p_{0}\right\}=\frac{1}{\mathrm{~g}} \int_{-\pi / 2}^{\pi / 2} \int_{0}^{p_{0}(\varphi, t)} H(m, v, \eta ; \varphi, p) a^{2} \cos \varphi \mathrm{d} p \mathrm{~d} \varphi+\int_{-\pi / 2}^{\pi / 2} p_{0}(\varphi, t) a^{3} \cos \varphi \mathrm{d} \varphi$,

where

$$
H(m, v, \eta ; \varphi, p) \doteq \frac{1}{2}\left(\frac{m}{a \cos \varphi}-\Omega a \cos \varphi\right)^{2}+\frac{1}{2} v^{2}+h(\eta, p)
$$

and

$$
p_{0}(\varphi, t) \doteq g \int_{a}^{a+\Delta r(\varphi, t)} \rho \mathrm{d} r>0
$$

is the (time-dependent) surface pressure, determined dynamically from the lower boundary condition $\mathrm{D} \Phi / \mathrm{D} t=0,(2.1 c)$ and $(2.1 e)$ :

$$
\frac{\partial p_{0}}{\partial t}+\frac{v}{a} \frac{\partial p_{0}}{\partial \varphi}=-\int_{0}^{p_{0}(\varphi, t)} \frac{1}{a \cos \varphi} \frac{\partial}{\partial \varphi}(v \cos \varphi) \mathrm{d} p .
$$

For convenience, let us rescale $\mathscr{H} \doteq \mathrm{g} \overline{\mathscr{H}} / a^{2}$.

The conservation properties of this system can be demonstrated with the following generalization of the transport theorem. For any quantity $I$ satisfying

$$
\frac{\mathrm{D}}{\mathrm{D} t} I=S
$$

(e.g. take $I=u, v$, or $\eta$ ) and any arbitrary function $f=f(I, p)$, we see that

$$
\begin{aligned}
\frac{\mathrm{d}}{\mathrm{d} t} & \int_{-\pi / 2}^{\pi / 2} \int_{0}^{p_{0}} f(I, p) \cos \varphi \mathrm{d} p \mathrm{~d} \varphi \\
& =\int_{-\pi / 2}^{\pi / 2} \int_{0}^{p_{0}}\left[f_{I}(S-v \cdot \nabla I)+f_{p}\left(\frac{\mathrm{D} p}{\mathrm{D} t}-\boldsymbol{v} \cdot \nabla p\right)\right] \cos \varphi \mathrm{d} p \mathrm{~d} \varphi+\int_{-\pi / 2}^{\pi / 2} f \frac{\partial p_{0}}{\partial t} \cos \varphi \mathrm{d} \varphi \\
& =\int_{-\pi / 2}^{\pi / 2} \int_{0}^{p_{0}}\left[f_{I} S+f_{p} \frac{\mathrm{D} p}{\mathrm{D} t}-\nabla \Delta(f v)\right] \cos \varphi \mathrm{d} p \mathrm{~d} \varphi+\int_{-\pi / 2}^{\pi / 2} f \frac{\partial p_{0}}{\partial t} \cos \varphi \mathrm{d} \varphi \\
& =\int_{-\pi / 2}^{\pi / 2} \int_{0}^{p_{0}}\left(f_{I} S+f_{p} \omega\right) \cos \varphi \mathrm{d} p \mathrm{~d} \varphi .
\end{aligned}
$$

In the last line we used the divergence theorem and the fact that in pressure coordinates the normal component of the three-dimensional velocity $v$ on the lower 
surface is

$$
\boldsymbol{v} \cdot\left(\hat{\boldsymbol{p}}-\frac{1}{a} \frac{\partial p_{0}}{\partial \varphi} \hat{\boldsymbol{\varphi}}\right)=\omega_{0}-\frac{v}{a} \frac{\partial p_{0}}{\partial \varphi}=\frac{\partial p_{0}}{\partial t}
$$

where $\omega_{0} \doteq \mathrm{D} p_{0} / \mathrm{D} t$, the value of $\omega$ on the lower surface, is determined by vertically integrating (2.1c).

For example, one may readily verify that $\mathrm{d} \mathscr{H} / \mathrm{d} t=0$ by repeated application of (2.20) together with $(2.8 b)$ and the result

$$
\begin{aligned}
-\int_{-\pi / 2}^{\pi / 2} \int_{0}^{p_{0}} \frac{v}{a} \Phi_{\varphi} \cos \varphi \mathrm{d} p \mathrm{~d} \varphi & =-\int_{-\pi / 2}^{\pi / 2} \int_{0}^{p_{0}} \Phi \frac{\partial \omega}{\partial p} \cos \varphi \mathrm{d} p \mathrm{~d} \varphi \\
& =-\int_{-\pi / 2}^{\pi / 2} a g \omega_{0} \cos \varphi \mathrm{d} \varphi-\int_{-\pi / 2}^{\pi / 2} \int_{0}^{p_{0}} \frac{\omega}{\rho} \cos \varphi \mathrm{d} p \mathrm{~d} \varphi
\end{aligned}
$$

which follows from $(2.1 \mathrm{c})$ and (2.1e). The first term on the right-hand side of (2.22) is seen to vanish upon considering the volume integral of $(2.1 \mathrm{c})$. Note that the surface term of $\mathscr{H}$ is just a constant proportional to the total mass, as can be seen by setting $f=1$ in (2.20).

In the special case where $S=0$ and $f=f(I),(2.20)$ implies that the densityweighted volume integral of an arbitrary function of any Lagrangian invariant $I$ is constant in time:

$$
\frac{\mathrm{d}}{\mathrm{d} t} \int \rho f(I) d V=\frac{1}{g} \frac{\mathrm{d}}{\mathrm{d} t} \int_{-\pi / 2}^{\pi / 2} \int_{0}^{p_{0}} f(I) a^{2} \cos \varphi \mathrm{d} p \mathrm{~d} \varphi=0 .
$$

In particular, the volume integral

$$
\mathscr{C}\left\{m, v, \eta, p_{0}\right\}=\int_{-\pi / 2}^{\pi / 2} \int_{0}^{p_{0}} C(m, \eta, q) \cos \varphi \mathrm{d} p \mathrm{~d} \varphi
$$

of any arbitrary function $C$ of the Lagrangian invariants $m, \eta$, and $q$ will be conserved: $\mathrm{d} \mathscr{C} / \mathrm{d} t=0$.

\section{Zonal equilibrium}

Consider an equilibrium state representing steady baroclinic zonal flow:

$$
u=U(\varphi, p), \quad v=0, \quad \eta=N(\varphi, p),
$$

where $U$ and $N$ have continuous first derivatives. For this equilibrium the lower boundary condition $\mathrm{D} \Phi / \mathrm{D} t=0$ is equivalent to the condition $\omega=0$ on the surface; (2.1c) then implies that $\omega=0$ everywhere. Equations (2.1a) and (2.1d) are therefore satisfied trivially, while $(2.1 b)$ becomes

$$
U^{2} \tan \varphi+2 \Omega U a \sin \varphi=-\Phi_{\varphi} .
$$

Upon differentiating this result with respect to $p$ and using the hydrostatic equation (2.1e), we obtain the thermal-wind relation

$$
2(U \tan \varphi+\Omega a \sin \varphi) U_{p}=-\Phi_{\varphi p}=-\Phi_{p \varphi}=-\left.\frac{1}{\rho^{2}} \frac{\partial \rho}{\partial \varphi}\right|_{p}=-\frac{\rho_{\eta}}{\rho^{2}} N_{\varphi},
$$

where $\rho$ and $\rho_{\eta}$ are evaluated at $(N, p)$. In terms of the total equilibrium angular momentum

$$
M(\varphi, p) \doteq U(\varphi, p) a \cos \varphi+\Omega a^{2} \cos ^{2} \varphi,
$$


the above relation may be written as

$$
\frac{2 \tan \varphi}{a \cos \varphi} M U_{p}=-\frac{\rho_{\eta}}{\rho^{2}} N_{\varphi} .
$$

Any fiow of the form (3.1) that obeys (3.5) will consequently satisfy the governing equations (2.1).

It will prove convenient to introduce the equilibrium potential temperature $\Theta(N)>0$ and potential vorticity

$$
Q \doteq-\frac{g}{c_{p}}\left(\zeta_{\mathrm{abs}} N_{p}+\frac{1}{a} U_{p} N_{\varphi}\right)=\frac{g}{c_{p} a^{2} \cos \varphi} \frac{\partial(M, N)}{\partial(\varphi, p)},
$$

where $\zeta_{\text {abs }}$ is the radial component of the absolute vorticity:

$$
\zeta_{\mathrm{abs}} \doteq \frac{U}{a} \tan \varphi-\frac{1}{a} U_{\varphi}+2 \Omega \sin \varphi=-\frac{M_{\varphi}}{a^{2} \cos \varphi} .
$$

One sees from (3.6) that whenever $Q$ is sign-definite, a single-valued transformation from $(\varphi, p)$ to $(M, N)$ will exist. However, we will soon see that our stability criteria require that $Q=0$ at the equator. Let us restrict our attention to equilibrium flows where $Q$ vanishes only on the equator and has a different sign in each hemisphere. One can then construct inverse functions $\varphi(M, N, Q)$ and $p(M, N, Q)$ that depend only on $M, N$, and the sign of $Q$. The latter dependence uniquely identifies the appropriate branch of the equilibrium profiles.

We wish to apply the so-called energy-Casimir method (Fjørtoft 1950; Kruskal \& Oberman 1958; Arnol'd 1965, 1966; Holm et al. 1985; see also Shepherd 1990, §6) to obtain stability criteria for this equilibrium flow. In the absence of dissipation, it is well known that the fully compressible (non-hydrostatic) hydrodynamical equations may be written within a non-canonical Hamiltonian framework in which the cosymplectic operator $\boldsymbol{J}$ is degenerate (Morrison \& Greene 1980); i.e. the phase-space variable $\boldsymbol{u}$ evolves in time according to

$$
\frac{\partial}{\partial t} \boldsymbol{u}=\boldsymbol{J} \frac{\delta \mathscr{H}}{\delta \boldsymbol{u}},
$$

such that $\operatorname{det} \boldsymbol{J}=0$. (The functional derivative $\delta \mathscr{H} / \delta \boldsymbol{u}$ is defined in the usual way as the coefficient of the lowest-order term of the integrated power series expansion in $\delta \boldsymbol{u}$ of an induced perturbation $\delta \mathscr{H}$.) In addition to the invariants associated with symmetries of the Hamiltonian, such systems conserve additional quantities, known as Casimirs, that have gradients lying in the kernel of $J$. While stationary solutions of the canonical Hamiltonian equations arise as extremals of the Hamiltonian, those of non-canonical systems correspond to extremizations constrained to surfaces defined by the invariance of the Casimirs. Although we will not prove that $\mathscr{H}$ provides a Hamiltonian for our constrained dynamical system or that $\mathscr{C}$ is indeed a Casimir, the energy-Casimir method is still applicable, provided we demonstrate that the resulting conditional extremals are steady solutions of the equations of motion. The stability of these equilibria can then be examined by considering second-order variations of the invariant $\mathscr{H}+\mathscr{C}$.

Let us therefore seek equilibria corresponding to conditional extremals of $\mathscr{H}$ on surfaces of constant $\mathscr{C}$, appealing to the method of Lagrange multipliers. Because one of the dynamical variables, $p_{0}$, appears in the limits of integration, the first variation of $\mathscr{H}+\mathscr{C}$ includes both volume and surface contributions (as is conventional, we 
absorb the multipliers into the function $C$ ):

$$
\begin{aligned}
0 & =\delta(\mathscr{H}+\mathscr{C}) \\
= & \int_{-\pi / 2}^{\pi / 2} \int_{0}^{p_{0}}\left[\left(H_{m}+C_{m}\right) \delta m\right. \\
& \left.+\left(H_{\eta}+C_{\eta}\right) \delta \eta+H_{v} \delta v+C_{q} \delta q\right] \cos \varphi \mathrm{d} p \mathrm{~d} \varphi \\
& +\int_{-\pi / 2}^{\pi / 2}[H+C+a g]_{p=p_{0}} \delta p_{0} \cos \varphi \mathrm{d} \varphi .
\end{aligned}
$$

We use this condition to determine the as yet unspecified function $C$.

First, the condition $C_{q}=0$ can be satisfied by choosing $C_{q}=0$ in each region of the flow where $Q$ is of definite sign and imposing the matching conditions

$$
\begin{aligned}
& \lim _{Q \rightarrow 0^{+}} C(M, N, Q)=\lim _{Q \rightarrow 0^{-}} C(M, N, Q)=C(M, N, 0), \\
& \lim _{Q \rightarrow 0^{+}} C_{q}(M, N, Q)=\lim _{Q \rightarrow 0^{-}} C_{q}(M, N, Q)=C_{q}(M, N, 0)
\end{aligned}
$$

to guarantee that $C_{q}=C_{q q}=0$ over the entire flow.

In each region of the flow in which $Q$ has a definite sign (and hence $\partial(M, N) / \partial(\varphi, p) \neq$ 0 ), the independent variables $\varphi$ and $p$ may then be expressed, via (3.1), in terms of $M$ and $N$. The equilibrium conditions

$$
H_{m}+C_{m}=0, \quad H_{\eta}+C_{\eta}=0
$$

can therefore be integrated to deduce that $H+C$ in each region is independent of $M$ and $N$, or equivalently, of $\varphi$ and $p$. Equations $(3.11 a, b)$ determine $C$ only to within an arbitrary constant. (Constant values of $C$ in (2.24) lead to invariants corresponding to the total mass.) In each region of sign-definite $Q$, let us choose this constant so that at equilibrium

$$
H+C=\frac{1}{2} v^{2}-a g=-a g
$$

this choice satisfies (3.10a) and ensures that the second integral in (3.9) vanishes. Equations (3.11) and (3.12) together with $H_{v}=0$ are sufficient to guarantee that $\delta(\mathscr{H}+\mathscr{C})=0$ in the equilibrium state.

Now

$$
H_{m}=\frac{m}{a^{2} \cos ^{2} \varphi}-\Omega, \quad H_{v}=v, \quad H_{\eta}=\frac{T}{\theta},
$$

upon using (2.8a). One finds for the equilibrium flow (3.1) that $H_{v}=0$ trivially, while evaluation of (3.11) leads to the prescriptions

$$
\begin{gathered}
C_{m}(M, N, Q)=-\frac{M}{a^{2} \cos ^{2} \varphi(M, N, Q)}+\Omega, \\
C_{\eta}(M, N, Q)=-\frac{T(N, p(M, N, Q))}{\Theta(N)} .
\end{gathered}
$$

Note that the condition

$$
\lim _{Q \rightarrow 0^{+}} \varphi(M, N, Q)=\lim _{Q \rightarrow 0^{-}} \varphi(M, N, Q)
$$

guarantees that both $C_{m}$ and $C_{\eta}$ are continuous across $Q=0$. Hence by (2.14), the matching condition (3.10b) is satisfied by (3.14).

These conditions determine the form of $C$ as a function of $M, N$, and $Q$; all other variables, including the (normally) independent variables $\varphi$ and $p$ must be treated 
as functions of the conserved quantities $M, N$, and $Q$ (to which they are related through the equilibrium profiles). In contrast, when $\mathscr{H}$ is functionally differentiated, the independent variables are held fixed.

It is important to establish the consistency of $(3.14 a, b)$. To do this, we will need to determine certain partial derivative relations. Equation (3.6) allows us to write

$$
\left.\frac{\partial \varphi}{\partial M}\right|_{N}=\frac{\partial(\varphi, N)}{\partial(M, N)}=\frac{\partial(\varphi, p)}{\partial(M, N)} \frac{\partial(\varphi, N)}{\partial(\varphi, p)}=\frac{g}{c_{p} a^{2} \cos \varphi} Q^{-1} N_{p}
$$

and

$$
\left.\frac{\partial p}{\partial M}\right|_{N}=\frac{\partial(p, N)}{\partial(M, N)}=\frac{\partial(p, \varphi)}{\partial(M, N)} \frac{\partial(p, N)}{\partial(p, \varphi)}=-\frac{g}{c_{p} a^{2} \cos \varphi} Q^{-1} N_{\varphi}
$$

In a similar manner one finds that

$$
\left.\frac{\partial \varphi}{\partial N}\right|_{M}=-\frac{g}{c_{p} a^{2} \cos \varphi} Q^{-1} M_{p}=-\frac{g}{c_{p} a} Q^{-1} U_{p}
$$

and

$$
\left.\frac{\partial p}{\partial N}\right|_{M}=\frac{g}{c_{p} a^{2} \cos \varphi} Q^{-1} M_{\varphi}=-\frac{g}{c_{p}} Q^{-1} \zeta_{\mathrm{abs}}
$$

upon using (3.7).

Now, $C_{\eta}$ in $(3.14 b)$ is to be regarded as a function of $M, N$, and $Q$ only, with the $M$-dependence entering through $p$. Hence

$$
C_{\eta m}=-\left.\left(\frac{T_{p}}{\Theta}\right) \frac{\partial p}{\partial M}\right|_{N} .
$$

Substitution of (2.9) and (3.17) into (3.20) yields

$$
C_{\eta m}=\frac{-B}{a \cos \varphi},
$$

where

$$
B \doteq \frac{g}{c_{p} a}\left(\frac{\rho_{\eta}}{\rho^{2}}\right) Q^{-1} N_{\varphi}
$$

Finally, upon using (3.14a), (3.18), (3.5), and (3.21) we verify that

$$
\begin{aligned}
C_{m \eta} & =-\left.\frac{2 \sin \varphi}{a^{2} \cos ^{3} \varphi} M \frac{\partial \varphi}{\partial N}\right|_{M}=\frac{2 g \tan \varphi}{c_{p} a^{3} \cos ^{2} \varphi} M Q^{-1} U_{p} \\
& =\frac{\mathrm{g}}{c_{p} a^{2} \cos \varphi}\left(\frac{-\rho_{\eta}}{\rho^{2}}\right) Q^{-1} N_{\varphi}=C_{\eta m} .
\end{aligned}
$$

This establishes the existence of a twice-differentiable function $C(m, \eta, q)$ satisfying (3.14).

\section{Formal stability theorem}

Since $\mathscr{H}+\mathscr{C}$ is conserved in time and $\delta(\mathscr{H}+\mathscr{C})=0$ by construction, the condition for formal stability (Holm et al. 1985) is that $\delta^{2}(\mathscr{H}+\mathscr{C})$ be sign definite (for arbitrary perturbations). Formal stability implies spectral or normal-mode stability as well as normed stability of the linearized equations. $\dagger$ Upon taking the variation of (3.9), we

$\dagger$ An interesting example demonstrating that this condition is not necessary for linear stability is discussed by Morrison (1993). 
find that

$$
\begin{aligned}
\delta^{2}(\mathscr{H}+\mathscr{C})= & \int_{-\pi / 2}^{\pi / 2} \int_{0}^{p_{0}}\left[\delta v^{2}+G_{m m} \delta m^{2}+2 G_{m \eta} \delta m \delta \eta+G_{\eta \eta} \delta \eta^{2}\right] \cos \varphi \mathrm{d} p \mathrm{~d} \varphi \\
& +\int_{-\pi / 2}^{\pi / 2}\left[2 G_{m} \delta m \delta p_{0}+2 G_{\eta} \delta \eta \delta p_{0}+\frac{\mathrm{d} G}{\mathrm{~d} p} \delta p_{0}^{2}\right]_{p=p_{0}} \cos \varphi \mathrm{d} \varphi
\end{aligned}
$$

where

$$
G(m, \eta, q ; \varphi, p) \doteq H(m, v, \eta ; \varphi, p)+C(m, \eta, q)-v^{2} / 2
$$

and

$$
\frac{\mathrm{d} G}{\mathrm{~d} p}=G_{m} m_{p}+G_{\eta} \eta_{p}+G_{p} .
$$

(Note that $G_{q}=0$.) No $\delta q$ contributions arise since the function $C$ was chosen to satisfy the matching conditions (3.10) as well as $C_{q}=0$ and $C_{q q}=0$.

For the equilibrium flow $G_{m}=G_{\eta}=0$ since $C$ was constructed to satisfy (3.11). Moreover, $(2.8 b)$ implies that

$$
G_{p}=1 / \rho \geqslant 0 .
$$

We then see from (4.3) and (4.1) that $\delta^{2}(\mathscr{H}+\mathscr{C})$ is sign definite (for arbitrary disturbances) if the symmetric matrix

$$
\boldsymbol{A} \doteq\left(\begin{array}{cc}
H_{m m}+C_{m m} & H_{m \eta}+C_{m \eta} \\
H_{\eta m}+C_{\eta m} & H_{\eta \eta}+C_{\eta \eta}
\end{array}\right)
$$

is positive definite almost everywhere. Note that the dependent variable $v$ is decoupled from $m$ and $\eta$.

In computing the Hessian (matrix of second derivatives) of $H$, we must hold the independent variables $\varphi$ and $p$ fixed. Since $H_{\eta m}=H_{m \eta}=0$, we obtain a diagonal matrix with the elements

$$
H_{m m}=\frac{1}{a^{2} \cos ^{2} \varphi}, \quad H_{\eta \eta}=0,
$$

upon using (2.11).

On the other hand, in evaluating the Hessian of $C$, all variables are to be differentiated with respect to $M$ or $N$. Using (3.14a), (3.16), (3.5), and (3.22) we calculate for the equilibrium flow,

$$
\begin{aligned}
C_{m m} & =-\frac{1}{a^{2} \cos ^{2} \varphi}-\left.\frac{2 \sin \varphi}{a^{2} \cos ^{3} \varphi} M \frac{\partial \varphi}{\partial M}\right|_{N} \\
& =\frac{1}{a^{2} \cos ^{2} \varphi}\left[-1-2 \tan \varphi M\left(\frac{g}{c_{p} a^{2} \cos \varphi}\right) Q^{-1} N_{p}\right] \\
& =\frac{1}{a^{2} \cos ^{2} \varphi}\left(-1+\frac{g}{c_{p} a} \frac{\rho_{\eta}}{\rho^{2}} Q^{-1} \frac{N_{\varphi}}{U_{p}} N_{p}\right) \\
& =\frac{B N_{p} / U_{p}-1}{a^{2} \cos ^{2} \varphi} .
\end{aligned}
$$


Next, we find from $(3.14 b),(2.9),(2.11),(3.19)$, and (3.22) that

$$
C_{\eta \eta}=-\left(\frac{T}{\Theta}\right)_{\eta}-\left.\frac{T_{p}}{\Theta} \frac{\partial p}{\partial N}\right|_{M}=\left(\frac{-\rho_{\eta}}{\rho^{2}}\right) \frac{g}{c_{p}} Q^{-1} \zeta_{\mathrm{abs}}=-\frac{B a \zeta_{\mathrm{abs}}}{N_{\varphi}} .
$$

Note that (3.6) can be used to eliminate $\zeta_{\mathrm{abs}}$ from $(4.7 \mathrm{~b})$ to obtain

$$
C_{\eta \eta}=\frac{B U_{p}}{N_{p}}\left(\frac{c_{p} a Q}{g U_{p} N_{\varphi}}+1\right)
$$

The condition $H_{m m}+C_{m m}>0$ is seen to be equivalent to

$$
B N_{p} / U_{p}>0
$$

while the condition $H_{\eta \eta}+C_{\eta \eta}>0$ may be written, recalling (3.7), as

$$
\rho_{\eta} Q M_{\varphi}>0 \text {. }
$$

Upon using (4.6), (4.7a), (4.8), and (3.21), we can express the condition $\operatorname{det} \boldsymbol{A}>0$ as

$$
\frac{B^{2}}{a^{2} \cos ^{2} \varphi}\left(\frac{c_{p} a Q}{g U_{p} N_{\varphi}}\right)>0,
$$

or, after using (3.22), as $\rho_{\eta} B U_{p}>0$. Together with (4.9), this result implies

$$
\rho_{\eta} N_{p}>0 \text {. }
$$

Note that the thermal-wind relation (3.5) allows us to rewrite (4.11) as

$$
-\rho_{\eta} f M Q / \Omega>0
$$

in terms of the Coriolis parameter $f \doteq 2 \Omega \sin \varphi$. The three criteria given by (4.10), (4.12), and (4.13) are not independent of each other: the determinant condition (4.13) along with either of the other two inequalities implies the third one.

Given any equation of state for which $\rho_{\eta}<0 \dagger$ (e.g. for a perfect gas $\rho_{\eta}=-\rho /\left(c_{p} \theta\right)$ ), these criteria reduce to

$$
-Q M_{\varphi}>0, \quad \frac{\partial \Theta}{\partial r}>0, \quad \frac{f M Q}{\Omega}>0 .
$$

It is instructive to compare the first criterion, which is equivalent to $Q \zeta_{\mathrm{abs}}>0$, with Rayleigh's condition for the stability of a cylindrical vortex to axisymmetric perturbations in an incompressible non-rotating fluid (Rayleigh 1916):

$$
u \zeta \doteq \frac{u}{r} \frac{\partial}{\partial r}(r u)>0 .
$$

We recognize the second criterion as the usual condition for static stability, while the third criterion is the result previously obtained by Stevens (1983). The three criteria are analogous to those found in the $f$-plane Boussinesq case (Cho et al. 1993).

As already noted, the criteria $(4.14 a-c)$ need only hold almost everywhere in order to prove formal stability. (In fact, it is clear that the left-hand side of $(4.14 c)$ will necessarily vanish at the equator (where $f=0$ ) and at the poles (where $M=0$ ).) Recall that we restricted attention to equilibrium flows where $Q$ is sign-definite in each hemisphere and changes sign across the equator. It is easy to see that if $Q$ changed sign anywhere except at the equator, the stability criteria could not be satisfied. For,

$\dagger$ There are fluids (e.g. water between 0 and $4^{\circ} \mathrm{C}$ ) with a positive $\rho_{\eta}$ : since $\partial \rho /\left.\partial T\right|_{p}=\rho_{\eta} \eta_{s} \partial s /\left.\partial T\right|_{p}=\rho_{\eta} \Theta c_{p} / T$, one sees that $\partial \rho /\left.\partial T\right|_{p}$ and $\rho_{\eta}$ have the same sign. 
in order to satisfy (4.14a) and (4.14c) simultaneously, both $M_{\varphi}$ and $M$ would have to change sign where $Q$ changed sign; this would contradict the assumed continuity of $M$ in $\varphi$. One may combine (4.14a) and (4.14c) to obtain the condition $-f M M_{\varphi} / \Omega>0$, which within each hemisphere precludes the existence of a local extremum of $M$ in $\varphi$. Given that $M$ vanishes at the poles, it follows that $M$ must be single-signed, having a single extremum at the equator.

We thus determine two possible configurations for stability: one with $M / \Omega<0$ and $f Q<0$ and the other with $M / \Omega>0$ and $f Q>0$ (almost everywhere). The latter represents the most likely situation for a planetary atmosphere, since $M / \Omega>0$ at the equator provided $U(0, p) / \Omega>-a$. (This condition is easily met for the Earth's atmosphere: since $\Omega a=465 \mathrm{~m} \mathrm{~s}^{-1}$, a violation would require supersonic wind speeds!)

Equation (4.13) may be interpreted in terms of the Richardson number $R_{i} \doteq \mathscr{N}^{2} / U_{r}^{2}$, where $\mathscr{N}^{2} \doteq g^{2} \rho_{\eta} N_{p}$ is the square of the Brunt-Väisälä frequency (which may be expressed in the more familiar form $\mathscr{N}^{2}=g \Theta_{r} / \Theta_{0}$, where $\Theta_{0} \doteq-\rho(\partial \rho / \partial \theta)^{-1}$ is a characteristic potential temperature). Note that (4.12) implies $\mathscr{N}^{2}>0$, verifying that (4.12) corresponds to static stability even when $\rho_{\eta}<0$. With the help of (3.5), we rewrite (3.6) as

$$
\begin{aligned}
Q & =-\frac{1}{c_{p}}\left[g \zeta_{\mathrm{abs}} N_{p}-\frac{1}{a \rho} U_{r} N_{\varphi}\right] \\
& =-\frac{1}{c_{p}}\left[g \zeta_{\mathrm{abs}} N_{p}-\frac{1}{a g}\left(\frac{1}{\rho_{\eta}}\right) \frac{2 \tan \varphi}{a \cos \varphi} M U_{r}^{2}\right] \\
& =-\frac{1}{c_{p} g \rho_{\eta}}\left[\zeta_{\mathrm{abs}} \mathscr{N}^{2}-f\left(\frac{M}{\Omega a^{2} \cos ^{2} \varphi}\right) U_{r}^{2}\right] \\
& =-\frac{f \mathscr{N}^{2}}{c_{p} g \rho_{\eta}}\left[\frac{\zeta_{\text {abs }}}{f}-R_{i}^{-1}\left(1+\frac{U}{\Omega a \cos \varphi}\right)\right] .
\end{aligned}
$$

Hence (4.13) can be rewritten as

$$
\left.\frac{M}{\Omega}\left[\frac{\zeta_{\mathrm{abs}}}{f}-{R_{i}^{-1}}^{-1}+\frac{U}{\Omega a \cos \varphi}\right)\right]>0
$$

or

$$
\frac{\zeta_{\text {abs }}}{f}\left(1+\frac{U}{\Omega a \cos \varphi}\right)>R_{i}^{-1}\left(1+\frac{U}{\Omega a \cos \varphi}\right)^{2}>0,
$$

which leads to the stability criterion

$$
R_{i}>\frac{f}{\zeta_{\mathrm{abs}}}\left(1+\frac{U}{\Omega a \cos \varphi}\right)>0 .
$$

For the special case $U(\varphi, p)=U_{0}(p) / \cos \varphi$ one finds $\zeta_{\mathrm{abs}}=f ;(4.19)$ then reduces to

$$
R_{i}>1+\frac{U}{\Omega a \cos \varphi}>0 .
$$

\section{Finite-amplitude stability theorem}

From the exact invariants $\mathscr{H}$ and $\mathscr{C}$, let us construct the finite-amplitude disturbance invariant

$$
\mathscr{A} \doteq[\mathscr{H}+\mathscr{C}]\left(m, v, \eta, p_{0}\right)-[\mathscr{H}+\mathscr{C}]\left(M, 0, N, P_{0}\right)
$$


for the disturbed state defined by

$$
m \doteq M+m^{\prime}, \quad v \doteq v^{\prime}, \quad \eta \doteq N+\eta^{\prime}, \quad p_{0} \doteq P_{0}+p_{0}^{\prime} .
$$

This invariant is called the disturbance pseudoenergy (e.g. Shepherd 1990). If one defines

$$
\Delta \doteq \int_{0}^{p_{0}} G(m, \eta, q ; \varphi, p) \mathrm{d} p-\int_{0}^{P_{0}} G(M, N, Q ; \varphi, p) \mathrm{d} p
$$

one may express $\mathscr{A}$ in the compact form

$$
\mathscr{A}=\int_{-\pi / 2}^{\pi / 2}\left(\int_{0}^{p_{0}} \frac{1}{2} v^{2} \mathrm{~d} p+\Delta+a g p_{0}^{\prime}\right) \cos \varphi \mathrm{d} \varphi .
$$

To analyse finite-amplitude stability, we must extend the domain of the equilibrium profiles $M(\varphi, p)$ and $N(\varphi, p)$ to values of $p>P_{0}$ subject to the thermal-wind balance condition (3.5). This extension may be made in an arbitrary fashion, provided that the final stability criterion (5.19) holds over the extended domain of $M$ and $N$. (However, the details of the extension will affect the upper bound (5.22); one might be able to use this arbitrariness to reduce the bound as much as possible.) Bounds on the pseudoenergy can then be obtained by decomposing $\Delta$ into its volume and surface contributions: $\Delta=\Delta_{V}+\Delta_{S}$, where (Mu Mu, private communication, 1993)

$$
\begin{aligned}
& \Delta_{V} \doteq \int_{0}^{p_{0}}[G(m, \eta, q ; \varphi, p)-G(M, N, Q ; \varphi, p)] \mathrm{d} p, \\
& \Delta_{S} \doteq \int_{P_{0}}^{p_{0}} G(M, N, Q ; \varphi, p) \mathrm{d} p .
\end{aligned}
$$

Taylor's Remainder Theorem implies that for each value of $\varphi$ and $p$, there exists some $\widetilde{m} \in(M, m), \widetilde{\eta} \in(N, \eta)$ such that

$$
\begin{aligned}
\Delta_{V}= & \int_{0}^{p_{0}}\left[G_{m}(M, N, Q) m^{\prime}+G_{\eta}(M, N, Q) \eta^{\prime}\right. \\
& \left.+\frac{1}{2} G_{m m}(\widetilde{m}, \widetilde{\eta}, \widetilde{q}) m^{\prime 2}+G_{m \eta}(\widetilde{m}, \widetilde{\eta}, \widetilde{q}) m^{\prime} \eta^{\prime}+\frac{1}{2} G_{\eta \eta}(\widetilde{m}, \widetilde{\eta}, \widetilde{q}) \eta^{\prime 2}\right] \mathrm{d} p
\end{aligned}
$$

where $G(m, \eta, q) \equiv G(m, \eta, q ; \varphi, p)$. Similarly, the application of Taylor's Remainder Theorem to $\Delta_{S}$ as a function of $p_{0}$ about $P_{0}$ implies that

$$
\Delta_{S}=G\left(M, N, Q ; \varphi, p_{0}\right) p_{0}^{\prime}+\left.\frac{1}{2} \frac{\mathrm{d}}{\mathrm{d} p} G(M, N, Q ; \varphi, p)\right|_{p=\widetilde{p}_{0}} p_{0}^{\prime 2},
$$

for some $\widetilde{p}_{0} \in\left(P_{0}, p_{0}\right)$.

The linear terms of $\Delta_{V}$ vanish since for the equilibrium state $C$ obeys (3.11). The first term of (5.7) cancels the third term in (5.4), because of (3.12). These results reflect the fact that $\delta(\mathscr{H}+\mathscr{C})=0$ for the equilibrium flow. Again, no $\delta q$ contributions arise since the function $C$ was chosen to satisfy (3.10).

It will be convenient to decompose the pseudoenergy (5.4) into volume and surface contributions such that $\mathscr{A}=\mathscr{A}_{V}+\mathscr{A}_{S}$, where

$$
\mathscr{A}_{V} \doteq \frac{1}{2} \int_{-\pi / 2}^{\pi / 2} \int_{0}^{p_{0}(\varphi, t)}\left(v^{2}+I_{V}\right) \cos \varphi \mathrm{d} p \mathrm{~d} \varphi
$$




$$
\left.\mathscr{A}_{S} \doteq \frac{1}{2} \int_{-\pi / 2}^{\pi / 2} I_{S}\right|_{p=\tilde{p}_{0}} \cos \varphi \mathrm{d} \varphi .
$$

From (5.6) and (5.7) we identify

$$
\begin{aligned}
& I_{V} \doteq \widetilde{G}_{m m} m^{\prime 2}+2 \widetilde{G}_{m \eta} m^{\prime} \eta^{\prime}+\widetilde{G}_{\eta \eta} \eta^{\prime 2}, \\
& I_{S} \doteq \frac{\mathrm{d}}{\mathrm{d} p} G(M, N, Q ; \varphi, p) p_{0}^{\prime 2} .
\end{aligned}
$$

Here, a tilde is used to indicate that the corresponding function is evaluated at $(\widetilde{m}, \tilde{\eta}, \tilde{q} ; \varphi, p)$.

For the surface contribution, (4.3) and (4.4) imply that $I_{S}=p_{0}^{\prime 2} / \rho(N, p)$ since $G_{m}=$ $G_{\eta}=0$ when evaluated at the equilibrium flow. Thus,

$$
\mathscr{A}_{S}=\frac{1}{2} \int_{-\pi / 2}^{\pi / 2} \frac{p_{0}^{\prime 2}}{\rho\left(N\left(\varphi, \widetilde{p}_{0}\right), \widetilde{p}_{0}\right)} \cos \varphi \mathrm{d} \varphi \geqslant 0 .
$$

The following lemma can be used to bound the interior contribution $I_{V}$.

LEMMA 1. If $\alpha>0$ and $x$ and $y$ are real, then

$$
-\alpha|G| x^{2}-\alpha^{-1}|G| y^{2} \leqslant 2 G x y \leqslant \alpha|G| x^{2}+\alpha^{-1}|G| y^{2} .
$$

Proof. The two inequalities

$$
0 \leqslant\left(\alpha^{1 / 2} x \pm \alpha^{-1 / 2} y\right)^{2}=\alpha x^{2} \pm 2 x y+\alpha^{-1} y^{2}
$$

provide the bounds

$$
-\alpha|G| x^{2}-\alpha^{-1}|G| y^{2} \leqslant \pm 2|G| x y \leqslant \alpha|G| x^{2}+\alpha^{-1}|G| y^{2},
$$

which in turn imply (5.11).

The application of Lemma 1 to $(5.9 a)$ yields, for any positive function $\alpha \equiv \alpha(\varphi, p)$,

$$
I_{V} \geqslant\left(\widetilde{G}_{m m}-\alpha\left|\widetilde{G}_{m \eta}\right|\right) m^{\prime 2}+\left(\widetilde{G}_{\eta \eta}-\alpha^{-1}\left|\widetilde{G}_{m \eta}\right|\right) \eta^{\prime 2} .
$$

In order to prove finite-amplitude stability, we would ideally like to bound $I_{V}$ and $I_{S}$ from above and below by positive-definite quadratic forms. However this is problematical, because $G$ depends on $p$ as well as on $m, \eta$, and $q$. The latter dependencies are not difficult to handle since the variables are Lagrangian invariants, and thus their range of values is always the same as the range provided by the initial conditions. But the surface pressure $p_{0}$ is a dynamical quantity that can, in principle, take on any positive value whatsoever. Thus the $p$-dependence in $G$ is difficult to bound a priori. In particular, the surface density $\rho$ in (5.10) could conceivably approach either zero or infinity.

Thus we are unable to obtain the sort of strong convexity properties that were employed in the analysis of Cho et al. (1993). Nevertheless, we can still prove finiteamplitude stability. Upon noting that $H_{m \eta}=0$, we obtain from (5.14) and (4.6) the bound

$$
I_{V} \geqslant\left(\frac{1}{a^{2} \cos ^{2} \varphi}+\widetilde{C}_{m m}-\alpha\left|\widetilde{C}_{m \eta}\right|\right) m^{\prime 2}+\left(\widetilde{C}_{\eta \eta}-\alpha^{-1}\left|\widetilde{C}_{m \eta}\right|\right) \eta^{\prime 2}
$$

To bound $I_{V}$ in terms of the equilibrium quantities, we need to relate the range of 
the perturbed quantities appearing in (5.15) to the range of the equilibrium profiles. For each function $F \in\left\{C_{m m},\left|C_{m \eta}\right|, C_{\eta \eta}\right\}$ we suppose that

$$
\{F(\widetilde{m}, \widetilde{\eta}, \widetilde{q}):(\varphi, p) \in V\} \subseteq\{F(M, N, Q):(\varphi, p) \in V\} .
$$

This will be the case if the perturbation globally introduces no new values of the Lagrangian invariants $m$ and $\eta$ (the $q$-dependence serving only to identify, by its sign, the branch of the function). The bound (5.15) may then be used to obtain a lower estimate for $\mathscr{A}_{V}$. (In the most general situation, one needs to extend the domain of the functions $F$ to the new range of $\tilde{m}$ and $\tilde{\eta}$ without disturbing (3.11) and (5.15). Although this construction is always possible in the one-dimensional case (cf. Arnol'd 1966), it is not yet clear whether two-dimensional functions can always be extended in an analogous manner.)

Suppose now that there exists some positive constant $c$ that provides the global bound (for $-\pi / 2 \leqslant \varphi \leqslant \pi / 2$ and all $p \geqslant 0$ )

$$
\min \left(C_{m m}-\alpha\left|C_{m \eta}\right|, C_{\eta \eta}-\alpha^{-1}\left|C_{m \eta}\right|\right) \geqslant c>0 .
$$

Equations (4.6), (5.15), (5.16), and (5.17) then imply that the pseudoenergy contribution $\mathscr{A}_{V}$ is bounded by

$$
\mathscr{A}_{V} \geqslant \frac{1}{2} \int_{-\pi / 2}^{\pi / 2} \int_{0}^{p_{0}}\left[v^{\prime 2}+\left(\frac{1}{a^{2} \cos ^{2} \varphi}+c\right) m^{2}+c \eta^{2}\right] \cos \varphi \mathrm{d} p \mathrm{~d} \varphi .
$$

Upon substitution of (4.7) and (3.21), the criterion (5.17) appears as

$$
\min \left(\frac{B N_{p} / U_{p}-\alpha_{0}|B|-1}{a^{2} \cos ^{2} \varphi},-B \frac{a \zeta_{\mathrm{abs}}}{N_{\varphi}}-\alpha_{0}^{-1}|B|\right) \geqslant c>0
$$

where $\alpha_{0} \doteq \alpha a \cos \varphi$ and $B$ is given by (3.22).

One may now establish finite-amplitude stability for the flow in terms of the disturbance measure $\left\|\boldsymbol{x}^{\prime}\right\|_{c}$ of the vector $\boldsymbol{x}^{\prime} \doteq\left(m^{\prime}, v^{\prime}, \eta^{\prime}, p_{0}^{\prime}\right)$, where

$$
\left\|\boldsymbol{x}^{\prime}\right\|_{c}^{2} \doteq \frac{1}{2} \int_{-\pi / 2}^{\pi / 2} \int_{0}^{P_{0}+p_{0}^{\prime}}\left[v^{\prime 2}+\left(\frac{1}{a^{2} \cos ^{2} \varphi}+c\right) m^{\prime 2}+c \eta^{\prime 2}\right] \cos \varphi \mathrm{d} p \mathrm{~d} \varphi .
$$

Here, the surface pressure disturbance $p_{0}^{\prime}$ is related to the generalized potential temperature disturbance $\eta^{\prime}$ by

$$
p_{0}^{\prime} \doteq g \int_{a}^{a+\Delta r} \rho_{\eta} \eta^{\prime} d r
$$

Equations (5.18) and (5.10) imply that the evolution of the positive-definite quantity $\left\|\boldsymbol{x}^{\prime}\right\|_{\mathcal{c}}^{2}$ is bounded according to

$$
\left\|x^{\prime}(t)\right\|_{c}^{2} \leqslant \mathscr{A}_{V}(t) \leqslant \mathscr{A}(t)=\mathscr{A}(0) .
$$

Hence finite-amplitude disturbances are bounded for all time by a quantity depending only on the initial state of the flow; the bound decreases to zero as the initial disturbance tends to zero.

The disturbance measure defined by (5.20) is not actually a norm since it does not satisfy the property of homogeneity: in general, $\|\lambda \boldsymbol{x}\|_{\mathcal{c}} \neq|\lambda|\|\boldsymbol{x}\|_{c}$ for a scalar $\lambda$. However, (5.20) still provides a meaningful measure of disturbance size since it satisfies the important property that $\|\boldsymbol{x}\|_{c}^{2} \geqslant 0$, with equality occurring only when $\boldsymbol{x}=0$ almost everywhere (observe that $\eta^{\prime}=0$ implies $p_{0}^{\prime}=0$ ). 
Note that (5.17) requires that

$$
C_{m m}>\alpha\left|C_{m \eta}\right| \geqslant 0, \quad C_{\eta \eta}>\alpha^{-1}\left|C_{m \eta}\right| \geqslant 0 ;
$$

these inequalities in turn imply

$$
C_{m m}>\frac{\left|C_{m \eta}\right|}{C_{\eta \eta}}\left|C_{m \eta}\right|=\frac{C_{m \eta}^{2}}{C_{\eta \eta}} .
$$

Since for this case $H_{m m}>0$ and $H_{\eta \eta}=0$, it follows that the finite-amplitude stability criterion (5.17) implies the formal stability conditions $(4.14 a-c)$ obtained in $\$ 4$.

Let us examine the finite-amplitude stability criterion (5.19) in further detail. Upon using (3.6) to eliminate $\zeta_{\mathrm{abs}}$ we find that

$$
\frac{B N_{p}}{U_{p}}-1-c a^{2} \cos ^{2} \varphi \geqslant \alpha_{0}|B| \geqslant B N_{p}\left(\frac{c_{p} a Q}{g N_{\varphi}}+U_{p}\right)^{-1} .
$$

Dividing through by the positive expression on the right-hand side of the second inequality, we obtain

$$
\frac{c_{p} a Q}{g U_{p} N_{\varphi}} \geqslant\left(1+c a^{2} \cos ^{2} \varphi\right) \frac{1}{B N_{p}}\left(\frac{c_{p} a Q}{g N_{\varphi}}+U_{p}\right)>0 .
$$

We now use (3.5) to eliminate $U_{p}$ on the left-hand side. Upon recalling (3.22) we obtain a finite-amplitude generalization of (4.13):

$$
-\rho_{\eta}\left(\frac{f M Q}{\Omega}\right) \geqslant a \cos ^{2} \varphi\left(1+c a^{2} \cos ^{2} \varphi\right) \frac{\rho_{\eta} Q}{N_{p}}\left(\frac{c_{p} a Q}{g}+U_{p} N_{\varphi}\right)>0 .
$$

Analogues of the other formal stability conditions can also be obtained from (5.19), which requires that

$$
\begin{aligned}
-\rho_{\eta} Q \zeta_{\mathrm{abs}} & \geqslant \frac{c_{p} \rho^{2} Q^{2}}{g}\left(c+\alpha_{0}^{-1}|B|\right)>0, \\
\frac{B N_{p}}{U_{p}} & \geqslant 1+c a^{2} \cos ^{2} \varphi+\alpha_{0}|B|>0
\end{aligned}
$$

for some positive constant $c$. Equations (5.28a) and (5.28b) are finite-amplitude generalizations of (4.10) and (4.9), respectively. Substitution of (3.22) and (3.5) into $(5.28 b)$, together with (5.27), leads to the criterion

$$
\rho_{\eta} N_{p} \geqslant-\frac{c_{p} \rho_{\eta}}{g}\left(\frac{f M Q}{\Omega}\right)^{-1} Q^{2} a^{2} \cos ^{2} \varphi\left(1+c a^{2} \cos ^{2} \varphi+\alpha_{0}|B|\right)>0,
$$

which is a generalization of (4.12) to finite amplitude. Evidently these finite-amplitude conditions are all somewhat more restrictive than the corresponding formal stability conditions.

Let us emphasize that, although we have provided a bound on the evolution of the disturbance measure $\|\boldsymbol{x}\|_{c}$, we have not proven Liapunov stability. Because of the possibility that $\rho$ could approach arbitrarily close to zero as the flow evolves (e.g. in the presence of cavitation), there appears to be no way of providing an upper bound on the surface contribution to the pseudoenergy. This treatment differs from the approach taken by Holm et al. (1983) upon encountering a similar difficulty: since their calculation required a non-zero lower bound on the density, these authors simply made this a hypothesis. In contrast, the treatment here is dynamically self-consistent in that it requires no externally imposed assumptions on $\rho$. 


\section{Discussion}

We have applied the energy-Casimir method to the problem of symmetric stability of a compressible, hydrostatic planetary atmosphere with a general equation of state, including a proper treatment of the lower boundary condition. It has been shown that steady baroclinic zonal flows of the form (3.1) satisfy the variational principle (3.9), and are thus conditional extremals of the energy subject to constraints provided by a suitably constructed Casimir-like invariant. By considering the second variation of the corresponding energy-Casimir (or pseudoenergy) invariant functional about these equilibria, formal symmetric stability criteria have been derived: namely (4.10), (4.12), and (4.13). In the special case of a perfect gas these criteria reduce to $(4.14 a-c)$, as previously derived by Stevens (1983).

In comparison with the $f$-plane, Boussinesq analysis of symmetric stability of Cho et al. (1993), the present analysis exhibits two interesting mathematical features: multivalued inverse equilibrium profiles and a moving lower boundary in $p$-coordinates. The first of these is dealt with by allowing the Casimir-like functions to have an additional dependence on the sign of the potential vorticity, which identifies the branch of the function. The second feature leads to an explicit contribution from the lower boundary to the pseudoenergy. However this contribution is positive definite, and thus presents no problem for the formal stability analysis.

For finite-amplitude disturbances, on the other hand, the moving domain of integration presents significant difficulties because the range of values of $p$ cannot be bounded a priori. This means that strong convexity properties of the pseudoenergy, such as were employed by Cho et al. (1993), are not available in this case. In particular, Liapunov stability does not appear to be provable. Nevertheless, we show that the pseudoenergy is positive definite and that finite-amplitude stability conditions can be derived. By finite-amplitude stability we mean that a positive-definite measure of disturbance amplitude is bounded uniformly in time, with the bound decreasing to zero as the initial disturbance tends to zero (cf. Mu Mu et al. 1994); this result is expressed by (5.22). The finite-amplitude stability conditions imply formal stability and are expressible as generalizations of the formal symmetric stability conditions.

From the stability bound (5.22) we also obtain a rigorous upper bound on the kinetic energy of the motion in the meridional plane:

$$
\frac{1}{2} \int_{-\pi / 2}^{\pi / 2} \int_{0}^{p_{0}} v^{2}(t) \cos \varphi \mathrm{d} p \mathrm{~d} \varphi \leqslant \mathscr{A}(0) .
$$

Since (6.1) holds for any equilibrium for which $I_{V} \geqslant 0$ everywhere, it may be used to define a generalized available potential energy for a given baroclinic zonal flow (see Shepherd 1993): simply choose a stable equilibrium, compute $\mathscr{A}(0)$ for the resulting disturbance field (the difference between the initial and the equilibrium values), and then minimize $\mathscr{A}(0)$ over all possible stable equilibria. This construction of a generalized available potential energy was used by Cho et al. (1993) to provide rigorous upper bounds on the nonlinear saturation of symmetric instabilities. A similar calculation would certainly be possible in the present case.

The authors would like to thank $\mathrm{Mu} \mathrm{Mu}$, P. J. Kushner, and P. J. Morrison for their insight and numerous discussions related to this work, N. J. Balmforth for his comments, and one of the referees for pointing out an error in an early version of the manuscript. J.C.B. acknowledges support from a Natural Sciences and Engineering Research Council (NSERC) of Canada Postdoctoral Fellowship and from United 
States DoE contract No. DE-FG05-80ET-53088; T.G.S. acknowledges grant support from NSERC and from the Atmospheric Environment Service of Canada.

\section{REFERENCES}

ARNOL'D, V. I. 1965 Conditions for nonlinear stability of stationary plane curvilinear flows of an ideal fluid. Dokl. Akad. Nauk. SSSR 162, 975-978. English trans. Sov. Maths 6, 773-777 (1965).

ARNOL'D, V. I. 1966 On an a priori estimate in the theory of hydrodynamical stability. Izv. Vyssh. Uchebn. Zaved. Matematika 54, 3-5. English trans. Am. Math. Soc. Transl., Series 2, 79, 267-269 (1969).

Cho, H.-R., Shepherd, T. G. \& Vladimirov, V. A. 1993 Application of the direct Liapunov method to the problem of symmetric stability in the atmosphere. J. Atmos. Sci. 50, 822-836.

FJøRTOFт, R. 1950 Application of integral theorems in deriving criteria of stability for laminar flows and for the baroclinic vortex. Geofys. Publ. 17, 1-52.

Holm, D. D., Marsden, J. E., Ratiu, T. \& Weinstein, A. 1983 Nonlinear stability conditions and a priori estimates for barotropic hydrodynamics. Phys. Lett. 98A, 15-21.

Holm, D. D., Marsden, J. E., Ratiu, T. \& Weinstein, A. 1985 Nonlinear stability of fluid and plasma equilibria. Phys. Rep. 123, 1-116.

Kruskal, M. D. \& Oberman, C. R. 1958 On the stability of plasma in static equilibrium. Phys. Fluids 1, 275-280.

LoRenz, E. N. 1967 The Nature and Theory of the General Circulation of the Atmosphere. World Meteorological Organization, Geneva.

Morrison, P. J. 1993 Hamiltonian description of the ideal fluid. Tech. Rep. WHOI-94-12, Woods Hole Oceanographic Institution.

MorRison, P. J. \& GreENE, J. M. 1980 Noncanonical hamiltonian density formulation of hydrodynamics and ideal magnetohydrodynamics. Phys. Rev. Lett. 45, 790-794.

Mu Mu, Zeng QingCun, ShePherd, T. G. \& LiU Yongming 1994 Nonlinear stability of multilayer quasi-geostrophic flow. J. Fluid Mech. 264, 165-184.

RaYleigh, Lord 1916 On the dynamics of revolving fluids. Proc. R. Soc. Lond. A 93, 148-154.

ShePHeRD, T. G. 1990 Symmetries, conservation laws, and Hamiltonian structure in geophysical fluid dynamics. Adv. Geophys. 32, 287-338.

ShEPHERD, T. G. 1993 A unified theory of available potential energy. Atmos. Ocean 31, 1-26.

SteVENS, D. 1983 On symmetric stability and instability of zonal mean flows near the equator. $J$. Atmos. Sci. 16, 892-893. 